\title{
Reflexiones sobre la concepción del cuerpo y del movimiento para una educación integral de la primera infancia ${ }^{1}$
}

\author{
Elizabeth Gutiérrez ${ }^{2}$ \\ Colegio Leonardo Da Vinci, Bogotá, Colombia \\ elizagu72@gmail.com \\ Johanna Andrea Castillo ${ }^{3}$ \\ Secretaría de Integración Social "SDIS" \\ jacastilloc@gmail.com
}

1 Artículo de investigación derivado de la investigación para la tesis de Maestría en Desarrollo Cognitivo. TecVirtual- Tecnológico de Monterrey, México

2 Magister en Educación con énfasis en desarrollo Cognitivo, TecVirtual- Tecnológico de Monterrey, México

3 Fonoaudióloga, Facultad de Medicina de la Universidad Nacional de Colombia 
Tema Libre

\title{
Reflexiones sobre la concepción del cuerpo y del movimiento para una educación integral de la primera infancia
}

\section{Resumen}

El artículo propone a educadores del nivel inicial o preescolar, una reflexión sobre la consideración ideal que se debiera tener del niño como ser pisco-físico-social en crecimiento. Se fundamenta en estudios de neuropsicología del desarrollo humano, sobre los cuales se apoya toda propuesta educativa, y en las experiencias didácticas que han buscado llenar de significado la escolaridad preescolar en torno a la exploración del cuerpo, entendiendo el movimiento y la actividad motriz como elementos didácticos, expresivos y creativos, desde el punto de vista de la conciencia, las emociones y las relaciones. Con base en estos fundamentos, se analiza cómo se podrían incluir en la escolarización de la primera infancia, la corporeidad y su vivencia activa por medio del movimiento, dando a éstos cabida en el en el aula, por su implicación en el desarrollo del niño, así como valorando las respuestas de éste a los estímulos brindados.

Palabras clave: educación inicial, cuerpo, movimiento, expresión corporal, primera infancia.

\section{Reflections about the conception of body and movement for an integral education in the early childhood.}

\begin{abstract}
This article proposes to educators at the initial or pre-school level a reflection about an ideal about how the child should be construed in terms of its physical and psychosocial being during its development. This study is based on neurophysiologic studies on human development on which all the educational proposals rest and on the teaching experiences which experiences that have sought revitalize kindergarten schooling around the exploration of the body, understanding movement and motor activity as educational, creative, expressive elements and from the point of view of consciousness, emotions and relationships. Based on these foundations, the article discusses how corporeality and active experience through movement could be included in the education of early childhood in a classroom context as it influences the development of the child and as well as assessing its responses to the stimuli provided.
\end{abstract}

Keywords: Initial Education; body; motion; body language; early childhood

\section{Reflexões sobre a conceição do corpo e do movimento para uma educação integral da primeira infância.}

\section{Resumo}

O artigo propõe aos professores de nível inicial ou pré-escolar, uma reflexão sobre a perspectiva ideal que deveria se ter da criança como ser psico-físico-social em crescimento. Fundamenta-se em estudos da neuro psicologia do desenvolvimento humano, sobre os quais está apoiada toda proposta educativa, e nas experiências didáticas que buscaram encher de sentido a escolaridade pré-escolar em torno à exploração do corpo, compreendendo o movimento e a atividade motriz como elementos didáticos, expressivos e criativos, desde o ponto de vista da consciência, as emoções e as relações. Com base nestes fundamentos, analiza-se como poderiam ser incluídos, na escolarização da primeira infância, a corporeidade e sua vivência ativa por meio do movimento, dando-lhes um lugar na aula, por sua relevância no desenvolvimento da criança, assim como dando valor às respostas desta aos estímulos oferecidos.

Palavras chave: educação inicial; corpo; movimento; expressão corporal; primeira infância. 


\section{Introducción}

Desde los años setenta, la neurología y la psicología en su intención de comprender la manera de favorecer el desarrollo integral del hombre, encuentran que tal objetivo es posible si se beneficia un equilibrio armonioso entre los aspectos físico y psíquico, al que se podría llegar por medio de la integración de la experiencia corporal del contacto con el otro y con el medio ambiente (tabla 1). A la comprensión del proceso de desarrollo humano, Piaget integró el aspecto cognitivo, estableciendo que incluso el conocimiento está íntimamente ligado a la experiencia corporal, porque mediante ella el niño piensa, aprende, crea y afronta sus problemas. Al converger dichos estudios en el hecho de que durante los primeros seis años de vida, el ser evoluciona paralela e integralmente en los aspectos físico, afectivo y cognitivo, enfatizan que se debe dar gran importancia a la calidad de las experiencias y/o estímulos que se brinden al niño, porque la infancia es la base del crecimiento de la persona y marcará de manera indeleble su desarrollo.

\section{Teoría del desarrollo psico-afectivo y concepción de la experiencia corpórea}

Gesell

$1921 / 1940 / 1979$

Freud 1968

H. Wallon 1970

Ajuriaguerra

1978

Piaget 1923 -

1936
Teoría del desarrollo: La integración del desarrollo físico y mental se produce en secuencias definidas; siendo la conducta adaptativa la precursora de la inteligencia, al utilizar la experiencia para la solución de nuevos problemas. (Maganto, 1998: 666)

Psicoanálisis: Se llega a la organización psíquica entre los 5 y los 6 años; siendo la experiencia física, un elemento fundamental que permite la realización de la exploración y la identificación de sí mismo, en el desarrollo afectivo- relacional del niño (Vayer, 1973).

Psico-biología: La personalidad se estructura con relación a la función tónica y la función motriz; dos funciones estrictamente conectadas y dependientes entre ellas, la primera constituye constantemente el marco de la segunda, juntas son fundamentales para llegar al conocimiento-conciencia del propio esquema corporal (Vayer, 1973).

Psiquiatría infantil: los comportamientos psicomotores están siempre en función de las emociones, de la afectividad del sujeto.

Psicología del cognitivismo: el niño es resultado de la interacción de sus capacidades innatas con el medio que lo rodea. Al desarrollo del conocimiento lógico se llega cuando se adquiere la concepción espacio-temporal, que consiente interiorizar cognitivamente las relaciones topográficas de los objetos, concentrándose en la proximidad o lejanía de su ubicación (Ormrod, 2008)

TABLA 1. Teorías del desarrollo psico-afectivo en relación a la experiencia corpórea.

Fuente: Elaboración propia basada en revisión documental indicada, 2014. 
Con base en los avances científicos, y tomando conciencia de la veloz evolución tecnológica que modificó el modo de vivir, al igual que la forma de percibir la realidad y de relacionarse tanto en familia como a nivel social, se hizo evidente la necesidad de pensar un nuevo modo de concebir e integrar al niño en la sociedad, por lo cual se realizó ya en el año 2000, El Primer Simposio Mundial de Educación Inicial o parvularia del siglo XXI ${ }^{4}$, donde se establecieron los aspectos fundamentales que han guiado las políticas educativas para la primera infancia de nuestro tiempo:

- Los niños y las niñas del mundo tienen su derecho a una educación, nutrición y salud que aseguren su supervivencia, crecimiento y pleno desarrollo de sus potencialidades.

- Los primeros años de vida, incluyendo el período prenatal, son cruciales y decisivos para el desarrollo integral de la persona.

- La educación inicial es una etapa educativa con identidad propia y que hace efectivo el derecho a la educación.

- La educación inicial debe comenzar desde el nacimiento, con el fin de lograr el desarrollo integral de la persona y prevenir futuras dificultades .

- La educación inicial y el cuidado de la salud y nutrición tienen un efecto positivo en la reducción de las desigualdades socioeconómicas del aprendizaje.

- La familia es la primera e insustituible instancia en la educación y cuidado de los niños y niñas.

Estas indicaciones plantearon tanto a nivel familiar como institucional, el paso de una educación asistencial a un proceso educativo que reconoció al niño como ciudadano con plenos derechos, y por ende, merecedor de una educación adecuada a sus características y necesidades. También generaron una evolución en la intención educativa del preescolar, que se orientó a lograr el desarrollo integral de los niños. Uno de los aspectos centrales en este cambio de visión de la educación infantil es que busca una formación integral, aspecto que implica necesariamente facilitar el desarrollo físico-intelectual-emocional del infante en edad preescolar. Es decir, hace referencia a una experiencia educativa en el nivel inicial o preescolar donde necesariamente se considere el cuerpo del niño desde una perspectiva del ser humano, que debe desarrollar su conciencia y el cuidado de sí mismo en relación con el entorno. A partir de estas indicaciones, cada país ha continuado desarrollando un proceso de mejora, tanto a nivel legislativo como de calidad del servicio educativo para la infancia, haciendo presente la necesidad de crear modelos y perspectivas pedagógicas capaces de convertir la escolaridad de niños y

4 Primer Simposio Mundial de Educación Infantil (inicial o preescolar) de este nuevo siglo, realizado en Santiago de Chile, en el año 2000 
niñas, no solo en una situación de aprendizaje multidisciplinar, sino además en una experiencia de convivencia que propiciando la equidad y la participación facilite un camino para un ejercicio pleno de la ciudadanía.

\section{Fundamentos científicos sobre evolución humana: El cuerpo y movimiento como base del desarrollo integral del niño}

Los estudios del desarrollo humano continúan aún hoy su curso de evolución, reafirmando la unicidad cuerpo-mente (psicofísico) y la consideración de la actividad motriz como esencial en el proceso de desarrollo del niño. Conceptos de principios del siglo XX tales como la teoría de Piaget, que identifica la experiencia física como la primera etapa del conocimiento que desarrolla el individuo, ya que a través de las acciones inicialmente intuitivas se realizan las primeras interacciones con los objetos, sobre la base de los procesos de abstracción reflexiva mediante la cual adquiere la coordinación de los diferentes tipos de inteligencia. Hoy los explica la neurología con mayor claridad, describiendo la relación entre los sentidos y el cerebro.

La integración de las modalidades sensoriales se define como el proceso inconsciente del cerebro de organizar las informaciones o sensaciones que detectan los sentidos para su uso, dando significado a las experiencias al clasificar toda la información. Es la integración sensorial la que permite actuar o responder a experiencias o situaciones de modo significativo, lo que se denomina respuesta adaptativa, que está a la base del aprendizaje académico y de la conducta social. La integración sensorial se da gracias a que el cuerpo y la mente están coordinados por el sistema nervioso central (SNC), que conformado por el cerebro y la médula espinal, almacena informaciones de todo tipo (visuales, auditivas, perceptivas, emocionales). Dichas informaciones son útiles para el trabajo conjunto de todos los sistemas que conforman el cuerpo, incluso para el desarrollo y funcionamiento del aparato fonador, con el cual se expresan verbalmente las informaciones registradas gracias a la articulación del lenguaje, posible por las contracciones musculares implicadas en el habla.

El progresivo descubrimiento del propio cuerpo como fuente de sensaciones, la exploración de las posibilidades de acción y funciones corporales, constituirán experiencias obligatorias sobre las cuales ir construyendo el pensamiento infantil. De igual forma, las relaciones afectivas establecidas en situaciones de actividad motriz, y en particular mediante el juego, serán fundamentales para el crecimiento emocional. A la intervención desde la motricidad en la etapa de infantil le corresponde la tarea de dar respuestas a cuestiones planteadas u otras, como el excesivo sedentarismo de la infancia o la obesidad infantil (Gil-Madrona et al., 2008).

La capacidad sensorial corresponde a la necesidad del cuerpo para conocer y comprender su alrededor por medio del sistema nervioso, que se divide en periférico (SNP) y SNC. El cerebro se hace cargo del buen funcionamiento de la comunicación 
del cuerpo con la red externa, activando los nervios que llegan a la periferia (SNP) y por medio de las neuronas percibe toda la información sobre las características del ambiente y las condiciones internas de los órganos (figura 1).

De esta manera es que percibimos la sensibilidad, combinando los mensajes que entran con aquellos que salen, con el fin de poder estimar tamaños, distancias y otras características de los alrededores, de manera de estar atentos y reactivos ante cualquier situación. Las informaciones sensoriales y motoras se manejan y procesan en las mismas áreas del cerebro que actualmente se denominan áreas sensomotoras. Incluso, existe evidencia de que el cuerpo internamente simula cómo debe reaccionar ante las situaciones, aun en un momento en el cual no realiza una acción, pero la imagina.

Las conexiones entre las neuronas son las que nos permiten pensar, pero también expresarnos física y verbalmente. Por esto, la neurología, explica que es más importante contar con muchas conexiones (sinapsis) que con muchas neuronas, y que cada neurona establece muchas más sinapsis cuándo es estimulada, de aquí la importancia de tener activo el cerebro. También es oportuno pensar que mantener el cuerpo en actividad, entrena al cerebro en su capacidad perceptiva. Para el neurólogo Cacciavillani ${ }^{5}$, el movimiento puede tener el papel central en la acción desde la primera etapa de la vida.

1. La iniciativa de realizar una acción es tomada por las áreas anteriores de la corteza frontal que activan ba corteza pre-motora.

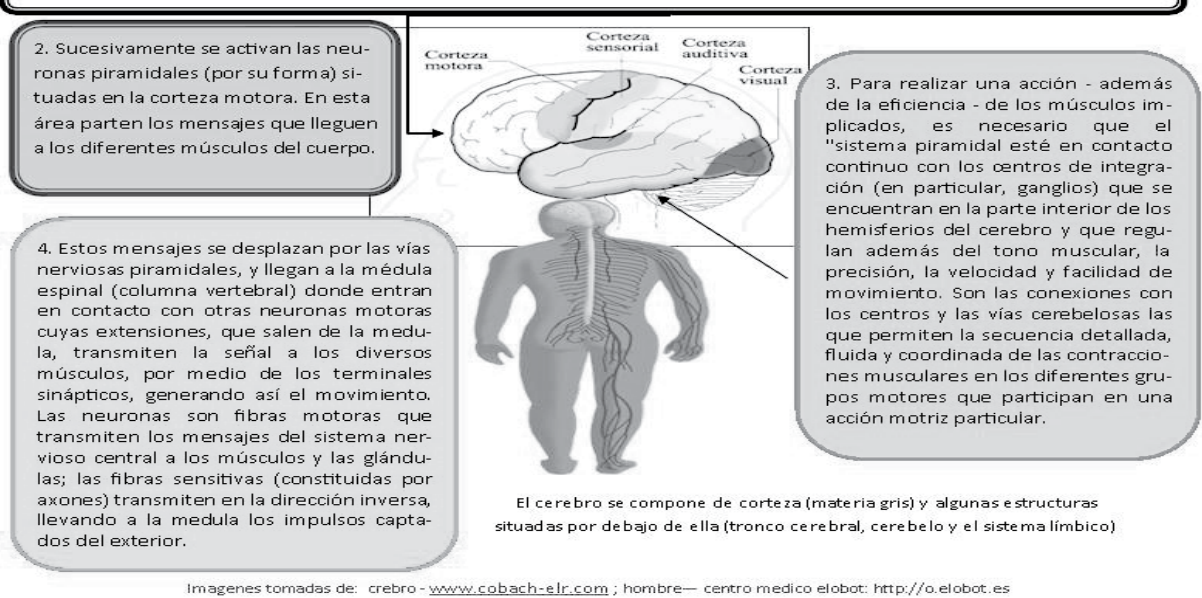

FIGURA 1. Relaciones del procesamiento del cerebro en relación a la actividad motora y sensoperceptiva. Fuente: Adaptación Elizabeth Gutiérrez basada en la revisión documental de Cacciacavilani, 2009 y Gallavotti, 1999. Gráficos: Cerebro tomado de www.cobach-eir.com y hombre tomado de centro médico elobot - http://o. elobot.es, 2011.

5 Cacciavillani, neurólogo italiano que se ocupa del desarrollo motor del niño y de la plástica neuronal. 
La interacción del cuerpo con el entorno implica la operatividad de las funciones tónica y motriz, gracias a la percepción de las sensaciones generadas en las diferentes posturas en relación con el entorno, activando así el conocimiento-conciencia que permite el aprendizaje y la comunicación inmediata. La operatividad por medio del conocimiento-conciencia del propio cuerpo, es lo que se define "esquema corporal" y corresponde a la imagen del propio cuerpo. Imagen, que según Wallon, se podría explicar cómo una intuición global de sí mismo o un conocimiento inmediato que se tiene del cuerpo, sea en posición estática o en movimiento, en relación a las diferentes partes entre ellas y sobre todo con el espacio y los objetos que lo circundan.

Wallon ${ }^{6}$ precisa que el esquema corporal "no es un dato inicial ni un entidad biológica o física, sino el justo resultado y la condición de las justas relaciones entre el individuo y el propio ambiente" (Vayer, 1973). El esquema corporal ha sido considerado el núcleo fundamental de la personalidad del cual depende el desarrollo equilibrado de los componentes afectivos, intelectuales y sociales de la persona. Esto implica que el desarrollo de la personalidad se da por etapas, en relación a dos instancias inseparables, la función tónica y la función motora, que en conjunto para cumplir su objetivos de hacer posible la comunicación, el conocimiento y la adaptación del ser al mundo, se apoyan en el tono muscular y el movimiento, elementos complementarios a la función neuromuscular.

Debido a que la experiencia física origina la experiencia sensorial, experiencia que permite la conciencia del esquema corporal, se resalta la importancia de consentir que el niño crezca en un entorno estimulante, porque la actividad de los 5 sentidos se produce primero a nivel físico, y posteriormente se convierte en experiencia sensorio-emocional. Esto hace evidente que el desarrollo intelectual y del lenguaje están relacionados con el desarrollo motor. Es decir, que la construcción de las estructuras cognitivas dependen de tres formas de conocimiento: el conocimiento psicofísico, el espacio-temporal y posteriormente el lógico-formal.

No obstante el cerebro mantenga toda su vida la plasticidad, esta está en su máximo nivel en los primeros años de desarrollo. $Y$ es en este periodo que grupos de neuronas deciden cuál será su tarea, si se ocuparan del lenguaje, la memoria - la actividad motora - o de cualquier otra función. Este hecho salió a la luz cuando se descubrió por casualidad que si se vendaba un ojo a un niño muy pequeño, por ejemplo a causa de una herida leve, él perdía la capacidad de ver por ese ojo. Esto sucedía porque las neuronas que debían transmitir las imágenes visuales a la corteza, no recibían ningún tipo de estímulo durante unas pocas semanas, abandonando al ojo que se tenía vendado para especializarse en otra función. Una vez que las células se encargan de otra función no hay manera de hacer que vuelvan a ocuparse de la anterior y el ojo es incapaz de ver, a pesar de que todas sus partes están funcionando perfectamente (Gallavotti, 1999).

6 Wallon Henri (1879-1962). Psicólogo y político francés. Como especialista en psicología de la infancia analizó la interdependencia de los factores biológicos y sociales en el desarrollo psíquico. 
Por otra parte, el conocimiento que el niño adquiere del mundo externo depende estrechamente de la relación que él tiene con el adulto, tal relación debe ser positiva para que el niño pueda reaccionar adecuadamente a la realidad circunstante. El análisis del desarrollo motor en concordancia con el desarrollo integral, hace evidente que el proceso de desarrollo de la inteligencia y el conocimiento de la persona, se lleva a cabo en etapas durante el crecimiento, dependiendo de la edad. No es casual que tanto a nivel pedagógico como a nivel neurológico se haya propuesto "el concepto de movimiento como sexto sentido". A nivel Pedagógico, la escuela Waldorf, según Baldassarre (2006) considera que el sentido del movimiento tan disturbado en nuestros días, corresponde a la capacidad de percibir todos los movimientos del cuerpo y las relaciones entre los movimientos, y es el que permite el refinamiento motor y a la capacidad de expresarse y de hablar bien. A nivel neurológico, Bertoz (1998, en Biferale 2009, 130), refiere dos significados del sentido del movimiento:

En el primero, considera el aspecto sensorial del movimiento como un sentido adicional a los cinco sentidos ya conocidos: un sexto sentido, por consiguiente, el movimiento sensorial. En el segundo introduce la capacidad de predicción del sistema motor, es decir, el sexto sentido que está dotado de función anticipatorio de lo que está por suceder en la realidad que nos circunda.

Siendo una realidad revelada a nivel científico que el gradual descubrimiento del cuerpo como principio de sensaciones, y la exploración de posibilidades de interacción y acción, constituyen experiencias inevitables sobre las que se construirá el desarrollo psicofísico y cognitivo del niño, otro aspecto a considerar es el lenguaje, entendido en sentido profundo como lo define Puyuelo (1998):

[...] una característica específicamente humana que desempeña importantes funciones a nivel cognitivo, social y de comunicación, que permite a los seres humanos hacer explicitas las intenciones, estabilizarlas, convertirlas en regulaciones muy complejas de acción humana y acceder a un plano positivo de autorregulación cognitiva y comportamental, al que no es posible llegar sin lenguaje, entonces la cognición humana se afecta según las experiencias corporales (motrices) que enfrenten los niños en su educación inicial (Puyuelo,1998)

\section{Experiencias educativas fundamentadas en el cuerpo, el movimiento libre y la actividad motriz como aspecto básico del desarrollo del niño.}

Desde mediados del siglo XX surgieron algunas experiencias educativas para ser aplicadas específicamente con niños, con la idea de llenar de significado la escolaridad infantil en torno a la vivencia del cuerpo y su exploración por medio del movimiento libre o de una actividad motriz pero no desde el punto de vista de la educación física. Estas se desarrollaron desde dos perspectivas: la concepción 
relacional ser-expresión-comunicación (tabla 2) y la concepción científica de la cognición humana (tabla 3). Estas perspectivas descansan sobre la base conceptual de que el cuerpo en la escuela debe ser vivenciado y experimentado en el movimiento, podría pensarse como un canal didáctico-comunicativo de vida, propusieron metodologías para realizar la actividad corporal con los niños y convergieron en un punto común, el ser humano necesita desarrollar la conciencia de sí mismo, de sus emociones y mayores relaciones porque estas son la base del aprendizaje, sea este psicofísico, cognitivo o socio-relacional.

\section{Experiencias educativas fundamentadas en el cuerpo y el movimiento libre desde la perspectiva relacional ser-expresión- comunicación.}

A principios del siglo XX, las nuevas concepciones ideológicas de liberación que buscaban la expresividad humana y la libertad en las artes, contrariamente a la mentalidad clásica y rígida de la época, conllevaron al surgimiento de nuevas prácticas corporales que apuntaban a mejorar las relaciones con el propio cuerpo teniendo como objetivo principal la desinhibición de la afectividad. Surgen entonces, movimientos de innovación en ámbito educativo y artístico de los años veinte hasta los treinta. Estos movimientos continuarán sus investigaciones solo después de las guerras mundiales y del periodo de la depresión socio-económica de los años 70, y llegaran a nuestros días. Entre los movimientos de innovación artística, son de notar grandes exponentes de la danza y de la música que se interesaron en la educación infantil (tabla 3). Considerándola fundamental para un cambio de paradigma educativo, generaron una fusión entre arte y educación escolar.

\begin{tabular}{|c|l|}
$\begin{array}{c}\text { Èmile } \\
\text { Dalcroze } \\
\mathbf{1 9 0 5}\end{array}$ & $\begin{array}{l}\text { Método para el aprendizaje musical denominado: rítmica, educación } \\
\text { rítmica o método pedagógico activo, que se desarrolla por medio de } \\
\text { la actividad didáctica Eutonía que implica el estudio del movimiento } \\
\text { (Vernia, 2012) }\end{array}$ \\
$\begin{array}{c}\text { Rudolf Von } \\
\text { Laban } \\
\mathbf{1 9 2 6 - 1 9 4 8}\end{array}$ & $\begin{array}{l}\text { Concepto de danza libre para un hombre libre. La principal } \\
\text { contribución de Laban en campo educativo es la creación de una } \\
\text { metodología práctica aplicada a la educación física, propuesta que } \\
\text { describe en sus textos Des Kindes Gymnastik und Tanz (gimnasia y } \\
\text { danza para el niño) 1926 y Modern Educational Dance (La danza } \\
\text { moderna educativa) 1948 y 1963. }\end{array}$ \\
\hline $\begin{array}{l}\text { Patricia } \\
\text { Stokoe 1950 }\end{array}$ & $\begin{array}{l}\text { Expresión corporal: por medio del método denominado } \\
\text { sensopercepción. Tiene como finalidad el desarrollo de la atención en } \\
\text { sí mismos, para encontrar el lenguaje corporal propio, "en el respeto } \\
\text { de las características y las habilidades individuales." Gubbay, M y } \\
\text { Kalmar, D.1985 }\end{array}$ \\
\hline
\end{tabular}

TABLA 2. Experiencias educativas fundamentadas en el cuerpo y el movimiento desde la perspectiva de la relación cuerpo-expresividad. Fuente: Elaboración propia, 2014. 
Siendo contrario a la enseñanza de la música por medio de métodos rígidos y mecánicos, y a partir de las dificultades que encontró en algunos de sus alumnos para el aprendizaje del solfeo, Delacroze ${ }^{7}$ integró en su docencia una serie de ejercicios corporales con los cuales recrear una imagen del sonido y del ritmo, instituyendo el método denominado "rítmica". Método que permite adquirir el sentido musical, por medio de las relaciones entre el ritmo interior (producto de la música) y el ritmo corporal. Si bien, Delacroze pensó inicialmente a la rítmica como método para niños a partir de los 6 años, pudo establecer que procuraba iguales beneficios a adultos, invidentes y personas en situaciones de discapacidad cognitiva. Entre los objetivos de la rítmica han sido citados: desenvolver y armonizar las funciones motrices y reglar los movimientos corporales en el tiempo y en el espacio (Langlade y Rey, 1983, 63); "el desarrollo del sentido musical en todo el cuerpo; el despertar de los instintos motores que dan conciencia de la noción de orden y de equilibrio; el aumento del desarrollo de las facultades imaginativas por el hecho del libre intercambio y de la unión intima entre el pensamiento y el movimiento corporal" (Baril 1987, 382).

Identificando el cuerpo como el mediador entre el sonido, el pensamiento y el sentimiento, Delacroze buscó integrar el cuerpo y la mente por medio del ritmo, utilizando como elementos el movimiento y la danza. Así favorecía con el ritmo la armonía del movimiento y la capacidad de adaptación y comprensión del sonido, al que se llega por un proceso de pensamiento. Delacroze consideraba que la educación musical debería ser obligatoria en la escuela, y logró integrar la rítmica en la educación escolar. A partir del estudio de las relaciones entre cuerpo-movimiento propuestas por Delacroze, posteriormente Laban $^{8}$ identificó en la relación del movimiento corporal los aspectos peso-espacio-tiempo-flujo, precisando en ellos las bases de su experimentación.

Rudolf Von Laban (Langlade y Rey, 1986; Kalmar, 2003; Copola, 2010) considerado uno de los creadores de la danza moderna, diferenció el movimiento deportivo del movimiento como arte, identificándolo no solo en su integridad emocional, sensitiva e intelectual, sino además, considerándolo un aspecto educativo. Propuso en el periodo de las guerras mundiales una metodología particular en la enseñanza de la educación física, en la cual la danza entra de lleno en el campo escolar. En su preocupación por una educación integral del niño, dejó de lado la perfección y ejecución de danzas sensacionales, focalizando el aspecto benéfico que la actividad creativa tiene sobre el alumno, insistiendo además en que "los niños, para lograr

7 Èmile Jaques- Dalcroze (1865-1950) Compositor, coreógrafo, actor y pedagogo suizo; considerado uno de los principales innovadores en la educación por sus estudios sobre la plástica como arte, influyendo ésta disciplina y en particular la danza moderna.

8 Laban, arquitecto, militar, filósofo y coreógrafo. Observó el movimiento en todos los aspectos de la vida, desde las artes marciales hasta evaluar las personas con enfermedades físicas y/o mentales. Sus teorías sobre movimiento y sus propuestas educativas, aún en nuestro días son vigentes, aplicables y de inspiración para estudiosos del tema. 
movimientos adecuados, han de pasar las etapas de exploración, experiencia y repetición". Así se aseguran formas personales de expresión "y cada niño crea su interpretación personal de sí mismo" (Langlade y Rey, 1986, 78). Zamorano (2010) reporta que "sus teorías tuvieron influencia en Inglaterra sobre los documentos oficiales de Educación Física en la enseñanza primaria denominados Moving and Growing (Movimiento y Crecimiento) y Planning the Programme (Planificando el Programa), publicados por el Ministerio de Educación y la Oficina Central de Información en Londres en 1952 y 1953 respectivamente"

Posteriormente en 1950 Patricia Stokoe ${ }^{9}$, inspirada en las propuestas educativas de Delacroze y Laban, así como en el trabajo de rehabilitación de Feldenkrais, integró estos planteamientos a sus propuestas y presentó la denominada "Expresión Corporal" (danza), estudio del movimiento como aspecto fundamental de la vida del ser humano, donde se experimenta el lenguaje del cuerpo con sus posibilidades de movimiento y quietud, sus gestos, posturas, tonos, destrezas y habilidades, organizadas en secuencias significativas como manifestación de la totalidad de la persona. Especificando los pilares de esta práctica, Stokoe dejó como aporte a la pedagogía de la danza, los contenidos que consideró aplicables en el proceso educativo: el área del cuerpo y el movimiento, el área de la comunicación y el área de la creatividad, que en la Expresión Corporal se enlazan en un estudio específico de la danza y el desarrollo de la persona, generando un elemento metodológico que guía esta área denominado sensopercepción. Stokoe experimentó la Expresión Corporal con los niños por varios años, dejando descritas sus consideraciones al respecto, de las cuales aclaraba que no daba recetas sino que contaba exclusivamente la experiencia vivida. En el texto "Expresión corporal" (1979. p.213), especifica concretamente cómo se desarrolla la actividad con los pequeños:

Una lección que tenga como propósito que el niño aprenda a servirse de su propio cuerpo considerándolo como otro instrumento de la Expresión total de sí mismo, pero que es necesario afinar, tal como se hace con un instrumento musical. A este perfeccionamiento corporal se llega poniendo al niño frente a la actividad, la cual tiene que variar según la edad y las capacidades de cada niño y de cada grupo. Se puede generar no obstante una regla general que es conveniente respetar cualquiera sea la edad de los alumnos: cada ejercicio o juego guiado debe iniciar de manera simple y fácilmente comprensible, y su nivel de dificultad se incrementará poco a poco. De frente a la superficialidad con la que muchas veces se practica el juego guiado, es necesario recordar que éste exige una organización precisa y dirigida a la enseñanza y que su planificación previa constituye el marco dentro del cual se podrá dar espacio libre a la inspiración individual.

9 Patricia Stokoe (1929-1996) Bailarina y pedagoga Argentina Stokoe, "fue una de las más importantes maestras de educación del arte y por el arte" (Kalmar 2004: 2). 


\section{Experiencias educativas fundamentadas en el cuerpo, el movimiento o la actividad motriz desde la perspectiva científica de la cognición humana}

En los años setenta, utilizando en manera sistemática el conocimiento de la neurofisiología, la genética y la psicología, anteriormente descritos, y con la intención de superar la concepción puramente biomecánica del movimiento, concluyendo que las prácticas deportivas y la educación física no son suficientes para una adecuada educación motriz del niño, surge en Francia la línea de investigación denominada psicomotricidad, propuesta de actividad motriz, práctica o enfoque educativo y terapéutico, basada en una concepción de la naturaleza humana integral de la actividad del cuerpo en interacción con la mente.

La psicomotricidad, desarrollada por Vayer, Le Boulch, Aucouturier y Lapierre, desde puntos de vista diferentes, en el campo educativo se diseña para la escuela elemental enfocada a integrar dinámicas adecuadas al crecimiento del niño, es decir, acordes a la concepción global, con la cual él adquiere el conocimiento (tabla 3). Partiendo de la consideración del cuerpo como esencia desde el punto de vista de la conciencia, las emociones y las relaciones, propusieron integrar la actividad motriz en la institución educativa infantil, haciendo evidente la necesidad de incluir nuevos elementos metodológicos que contemplaran un criterio participativo, en el cual el niño experimente conscientemente el espacio, los objetos y su capacidad relacional.
Vayer 1973 La educación corporal como principio dinámico de cada acción - Vayer/ educativa, fundamentada en la necesidad de un criterio global al
Destrooper problema psico-social de inadaptación infantil que ellos definen una 1976 esclerosis del diálogo.
Le Boulch - $\quad$ Propone la psicokinesis o psicocinética como método de educación 1977 al movimiento para niños hasta los 12 años.
Lapierre y Crean la psicomotricidad relacional y terapia psicoanalítica, con la Aucouturier cual consideran el movimiento como elemento insustituible en el desarrollo infantil.

TABLA 3. Línea francesa de educación psicomotriz. Fuente: Elaboración propia, 2014.

El concepto de criterio global de la educación sostiene que la experiencia física permite al niño concebir y comprender el entorno y en consecuencia los conceptos, poniendo la institución de educación inicial de frente a la importancia de reconocer el desarrollo motor y afectivo en el mismo nivel del intelectual, teniendo en cuenta la evidente necesidad de incluir nuevos elementos metodológicos, que motivarían a los maestros a enseñar todas las áreas con un criterio participativo, en el cual los menores experimenten conscientemente el proceso educativo intelectual y físico. 
Vayer $^{10}$ y Destrooper (1976) realizaron estudios de base neuro-psicológica con relación a sus propias experiencias psico-pedagógicas, en donde las primeras dan un significado a las segundas. En su estudio profundo sobre la inadaptación infantil que definen como una esclerosis del diálogo, concluyeron que el niño en su proceso de desarrollo e integración a la sociedad encuentra diferentes dificultades que pertenecen a dos tipos de situaciones que coexisten de hecho: una sobreestimulación (concerniente tanto a su naturaleza como a su forma) y una sobreestructuración de los estímulos. Plantearon que en alternativa a tal problema, la educación corporal debía ser el principio dinámico de cada acción educativa, convencidos de la necesidad de un criterio global al problema psico-social de gran actualidad. Buscando identificar el significado que pueden asumir los diferentes tipos de acciones y de comunicación corporal, presentes en el diálogo adulto-niño, sostuvieron que:

Los disturbios de la comunicación, para un joven, repercuten sobre todas las formas de contacto que este tiene con el mundo y muy pronto se convierte en un desadaptado al sistema de reglas que constituyen el punto central de la educación: al interior de la familia, del grupo de pares y de la escuela a la cual se ha encomendado. En la sociedad contemporánea, la escuela se coloca como medio, pero allí la comunicación es nuevamente y de la misma manera unidireccional. Considerando que la relación de ayuda del adulto con respecto al niño desadaptado sea válido solo si se trata de un criterio concebido y vivido en términos de desarrollo de la persona, es decir, en términos de existencia. Y un criterio global parecido se realiza solo si el niño hace propia aquella dimensión primaria del ser que es su cuerpo, porque la única relación y comunicación posible con el mundo es aquella corporal (Vayer y Destrooper, 1976)

Vayer y Destrooper evidenciaron cómo el desarrollo de las tecnologías de la comunicación en la sociedad tiene repercusiones en la comunicación que el niño establece con el ambiente que lo rodea; llegando a concluir que "el niño no tiene casi nada que buscar, ni que descubrir. El niño en cambio, tendría necesidad de orientar sus percepciones de una forma más coherente y de encontrar los medios que le permitan seleccionar y escoger entre la información que recibe, aquella que es verdaderamente útil al desarrollo de su persona" (Vayer y Destrooper, 1976).

Continuando una investigación basada en experimentos científicos en neurología y psico-somática, Le Boulch ${ }^{11}$ estructura una propuesta que pasa del movimiento espontáneo a un proceso psico-físico dirigido a producir una acción psico-motora, entendiendo por esta la capacidad de establecer una relación entre procesos

10 Pierre Vayer. Graduado en letras y ciencias humanas, docente de psicología y Doctor en Ciencias de la Educación; fue Director de investigación en el Instituto Piaget de Lisboa.

11 Jean Le Boulch (1924-2001). Profesor de educación física y deportes; estudió medicina para profundizar el movimiento humano, especializándose en Kinesiología (ciencia del movimiento), y también se gradúa en psicología. En 1966 crea la psicocinética, método de educación a través el movimiento, con la intención de que aplique también en psicomotricidad y fisioterapia. 
cognoscitivos y aprendizaje de las disciplinas deportivas, en una práctica consiente de esquemas motrices específicos en ambientes analógicos adaptados a cualquier edad, denominada psicokinesis o psico-cinética. Le Boulch (1981) la definió como la ciencia que "utiliza el movimiento humano en todas sus formas", con el objetivo de obtener una mejor "conciencia y aceptación de sí mismo, mejor adaptación de la conducta, verdadera autonomía y el inicio de una actitud de responsabilidad de sus propias acciones en el ámbito de la vida con relación a los otros". En el campo educativo, la psico-cinética introdujo las características de teóricos de la escuela activa, que apuntan a una educación basada en los intereses del niño, sosteniendo que debía ser "integrada en la educación de la escuela elemental, porque condiciona todos los aprendizajes preescolares y escolares. Estos no pueden ser conducidos a buen término si el niño no ha llegado a tomar conciencia de su cuerpo, a lateralizarse, a situarse en el espacio, a dominar el tiempo, si no ha adquirido una suficiente habilidad de coordinación de sus gestos y movimientos" (Le Boulch, 1983:27).

Otra línea de la investigación de la psicomotricidad es la denominada "psicomotricidad relacional" desarrollada por Lapierre ${ }^{12}$ y Aucouturier $^{13}$, por medio de la cual se busca sacar aquel aspecto de la personalidad que generalmente queda escondido "en la sombra, ignorado, negado, represo, culpabilizado; aquella dimensión enterrada de la ida afectiva con sus sentimientos, sus conflictos, sus ambivalencias, sus tensiones, sus angustias, la complejidad de sus contenidos proyectados y defensivos" (Lapierre, 2001). Estos aspectos los consideran como condicionantes del desarrollo y afirmación de la personalidad, que se expresa por medio de las capacidades relacionales e intelectuales, entre sus muchos aspectos. En relación al aspecto cognitivo, consideraron que de los procesos de adaptación motriz espontáneos originan los procesos de pensamiento, porque "el niño descubrirá un cierto número de nociones abstractas, que- será - capaz de utilizar como estructuras intelectuales mucho antes de poderlas expresar verbalmente" (Lapierre, 2001, p. 20). A partir de la experimentación de la "psicomotricidad relacional" Aucouturier crea una nueva corriente, para ocuparse específicamente de la motricidad en su aspecto expresivo:

Como lo define Aucouturier (2004): "la expresividad motriz es la manera que cada niño tiene de manifestar el placer de ser él mismo, de construirse de una manera autónoma y de manifestar el placer de descubrir y de conocer el mundo que lo rodea" (p. 130). Siempre, partiendo de la base de que un niño que se ha

12 Andrè Lapierre. Docente di Kinesiterapie a Paris desde 1946. Siendo docente de Rehabilitación infantil pone en discusión el método que tradicionalmente utiliza y concede mayor importancia a la calidad de las relaciones. Creó y dirigió (1968-1976) La Sociedad Francesa de Educación y Rehabilitación Psicomotriz, experiencia que lo llevó a iniciar una nueva etapa científica.

13 Bernard Aucouturier. Profesor de educación física especializada y miembro de la Academia Nacional de Medicina Prix de Thèse' del Ministerio de la Juventud y del Deporte de Francia. Se desempeñó como Director del centro de Educación física especializada y Profesor del centro Regional de Formación de Enseñantes para la adaptación y la integración escolar. 
apropiado de su cuerpo, tiene conciencia del mismo, y se ha movido y descubierto sus posibilidades, seguramente logrará ajustar sus posturas, movimientos y acciones a los requerimientos del entorno. (Martín, 2013, p. 76). En este sentido, el abordaje pedagógico de esta dimensión, se concibe desde su componente relacional y por lo tanto implica un proceso formativo mediado por la implicación personal y la toma de conciencia de su ser corporal: "Ios conceptos de respeto, acogida, escucha, comprensión serían sólo palabras sino se han vivido, experimentado, interiorizado" (Aucouturier, 2004, p. 202, en Martín, 2013, p.77)

Como una más reciente evolución del pensamiento psicomotor surgió la sociomotricidad de Parlebas ${ }^{14}$, quien cuestionó el objeto de la educación física, llegando a la conclusión que lo fundamental no es el movimiento sino la motricidad. Su intención era colocar la conducta motriz al centro de esta disciplina, y con esto, resaltar que está en juego toda la personalidad del practicante cuando actúa y mueve el su cuerpo (Parlebas, 2001, 79, En Cachadiña, 2004); en esto sentido, propone como objeto de estudio de esta área las conductas motrices que llevan a un tratamiento pedagógico. La sociomotricidad reporta a la dimensión social de la conducta, teniendo como elemento central el juego colectivo, por tanto, tal concepto lleva a Parlebas no solo a introducirse en el ámbito de la psico-sociología, sino también en la semiológica, entendida como aquella que estudia los sistemas de signos y los códigos de la comunicación.

La evolución de la psicomotricidad origina una reflexión pedagógica paralela y constante sobre la educación de la infancia, invitando a un análisis profundo sobre la concepción del cuerpo, el movimiento y la actividad motriz a nivel escolar, pero sin fines deportivos o terapéuticos. Entre los conceptos que aporta la psicomotricidad y los factores de desarrollo que favorece, se encuentran una mejor concepción de esquema corporal, conciencia y manejo de la respiración, desarrollo de la lateralidad y mejor manejo del equilibrio, gracias a actividades que permiten una relación vivencial espacio-temporal. Estos aportes han permitido que la educación motriz que se plantea en la escolaridad infantil se adapte a una visión integral e integrada del sujeto humano como unidad psicofísica, teniendo en cuenta el desarrollo de las condiciones: relacional, comunicativa, expresiva, operativa de la personalidad, como parte de la manera de ser, de actuar, de relacionarse como ser humano; superando la separación de la educación física del contexto total del proceso de enseñanza-aprendizaje en la escuela. Sin embargo, en lo que respecta al preescolar, aunque se han renovado los lineamientos, estructurándolos y enriqueciéndolos con planteamientos concretos que buscan integrar en sus propuestas los avances científicos en materia de desarrollo infantil, queda siempre una duda sobre cómo aplicar en la actividad docente tales lineamientos, debido a que los maestros en sus estudios pedagógicos y/o universitarios, cuentan con una a formación sólida a nivel teórico pero queda faltando siempre el aspecto didáctico practico de tales teorías.

14 Pierre Parlebas, doctor en Letras y Ciencias Humanas. 
Las prácticas de la psicomotricidad por el hecho de ser aplicada mayormente en los campo de fisioterapia y rehabilitación, a nivel educativo y de formación del docente de preescolar se integran como un estudio teórico pero no práctico, impidiendo esto que se puedan aplicar algunos conceptos y métodos en aula como enriquecimiento de un proceso escolar. Por tal motivo, es importante realizar un análisis sobre cómo se podrían aplicar la teoría sobre la consideración del cuerpo y su vivencia activa por medio del movimiento, en la escolarización de la infancia hoy, en todas las áreas y no solo en aquella motriz, dándole el valor que representa en la formación del niño por lo que implica en su desarrollo, así como valorando la respuesta de los pequeños a los estímulos brindados.

\section{Consideraciones de la concepción del cuerpo y el movimiento en la institución educativa del preescolar en Colombia}

La concepción de la educación infantil en Colombia ha evolucionado a lo largo de los años, paralelamente al desarrollo de la educación en el mundo, esto ha sucedido en dos aspectos: a nivel legislativo, en cuanto la educación preescolar se integra en las políticas de estado, y a nivel de servicio, en cuanto actualmente se considera fundamental como derecho ciudadano. A nivel legislativo, se pueden establecer dos grandes fases de este proceso, la reglamentación del servicio y el reconocimiento de un derecho ciudadano.

La fase legislativa de reglamentación del servicio, corresponde a aquella en la cual se define la enseñanza infantil como aquella dirigida a niños entre 5 y 7 años (Decreto 2101 del 39), se reglamentan los jardines infantiles y se indican los primeros lineamientos generales de educación preescolar (Decreto 1022 del 84) y se adoptan estrategias para promover el desarrollo infantil, reconociendo la importancia de mejorar sus condiciones de salud y nutrición (Plan Nacional SUPERVIVIR-1985), convergiendo finalmente en la creación de la Ley General de Educación (ley 115 de 1994), que en conformidad con la Constitución Política de Colombia declara como su objetivo: "la educación es un proceso de formación permanente, personal, cultural y social que se fundamenta en una concepción integral del niño, de su dignidad, de sus derechos y de sus deberes". Con base en ley 115 , se indican en 1998 los segundos lineamientos pedagógicos nacionales para el preescolar.

La fase legislativa de reconocimiento del preescolar como derecho ciudadano se puede establecer a partir de las indicaciones educativas para la primera infancia resultado del Primer Simposio Mundial de Educación Inicial del siglo XXI. Se verifica en el 2002 la Alianza por la Política Pública de Infancia y Adolescencia en Colombia, conformada por el Instituto Colombiano de Bienestar Familiar (ICBF), el Departamento Administrativo de Bienestar Social (DABS), el Centro Internacional de Educación y Desarrollo (CINDE), y otras 11 instituciones, algunas entre ellas internacionales. En 
el año 2006 se crea el Código de Infancia y adolescencia (Ley 1098), que en su artículo 29 establece "Derecho al desarrollo integral", reconociendo como ciclo vital la etapa de 0 a 6 años. Llegando más recientemente en el interés de ampliar la cobertura y mejorar la calidad del servicio, a crear dos leyes: el Plan Nacional de Desarrollo 2006-2010 dirigido a la infancia y la adolescencia y el acuerdo nacional de política pública para la educación inicial o preescolar denominado, "Colombia por la primera infancia" del 2007, suscrito por los Ministerios de Protección Social y Educación (MEN) junto con el ICBF. Sobre esta última, Castro (2013) identifica una carencia en campo aplicativo, que cabe notar, se ha presentado en todos los elementos legislativos anteriormente citados:

—En- La política pública [...] Colombia por la primera infancia [...] Llama poderosamente la atención, una vez leída su justificación, que los seis argumentos esgrimidos allí, los cuales abarcan desde el desarrollo humano, el carácter científico, el interés social y cultural, la naturaleza jurídica y política, otros ligados al contexto internacional y programático e inclusive uno de carácter ético; entre estos argumentos no se incluye aquel desde donde se generaron precisamente las condiciones de posibilidad para su enunciación, valga decir, el argumento pedagógico, que no puede diluirse en los seis invocados, o resolverlos con la ya familiar pero igualmente problemática consideración sobre su carácter implícito (Castro, 2013).

A nivel del servicio, es decir a la práctica educativa e institucional para el preescolar, en la actualidad la educación inicial es implementada y supervisada por el MEN, quien conjuntamente con las Secretarías de educación (SED) en cada municipio, indica y monitorea la aplicación de los recientes lineamientos pedagógicos y curriculares. Sin embargo, aunque en el lineamiento de preescolar se explique teóricamente las fases de desarrollo del niño y se relacionen con actividades motrices de las cuales se indican los objetivos a alcanzar, describiendo así como podría ser el Proyecto Educativo Institucional (PEI) de cada preescolar o escuela, la carencia de una línea didáctica de aplicación sigue siendo un vacío.

Analizando el caso de Bogotá, como referencia vanguardista del territorio nacional, se evidencia que con la intención de estructurar una propuesta que facilite el pleno desarrollo del niño, la Secretaría de Integración Social (SDIS), el MEN y la Alcaldía de Bogotá en el 2013, establecieron conjuntamente el Lineamiento Pedagógico y Curricular para la educación inicial del Distrito, en adelante Lineamiento del Distrito. Documento en el cual indican los aspectos de la dimensión humana que se deben integrar en la propuesta educativa: intelectual, moral, espiritual, social, afectiva y ética, en la adquisición de conocimientos en relación con la naturaleza, el conocimiento humano y cultural y el desarrollo del pensamiento lógico-matemático. 
En el Lineamiento del Distrito se considera la educación inicial en la institución educativa, denominada preescolar o escuela, como educación formal no en el sentido académico, sino en el netamente institucional y la define como "aquella que se dirige a los niños y niñas de la primera infancia (de edades entre 0 y 6 años), acorde con los requerimientos y demandas de su desarrollo, por lo cual es válida en sí misma y no solo como preparación para la educación formal" (Lineamiento, 2013, 9). Entre los lineamientos se especifica que la metodología a aplicar en preescolar implica cuatro pilares que son: el juego, el arte, la literatura y la exploración del medio; es decir, que éstos son los canales por medio de los cuales al realizar las actividades en aula se llegan a desarrollar las dimensiones y ejes de la educación inicial propuestos (tabla 4), sin embargo, falta una real reflexión sobre la didáctica y aspectos a valorar en tales procesos.

\begin{tabular}{|c|c|c|}
\hline Dimensiones & $\begin{array}{c}\text { Ejes - Campos de } \\
\text { trabajo }\end{array}$ & Desarrollos por fortalecer / a nivel general \\
\hline $\begin{array}{l}\text { Personal- } \\
\text { social }\end{array}$ & $\begin{array}{l}\text { Identidad } \\
\text { Autonomía } \\
\text { Convivencia }\end{array}$ & $\begin{array}{l}\text { - Identificar, descubrir, expresar y fortalecer } \\
\text { los vínculos afectivos. } \\
\text { - Desarrollar la seguridad en sí mismo }\end{array}$ \\
\hline Corporal & $\begin{array}{l}\text { Imagen, percepción y } \\
\text { conocimiento. } \\
\text { Movimiento como } \\
\text { medio de interacción. } \\
\text { La expresión y la } \\
\text { creatividad del cuerpo } \\
\text { en movimiento. }\end{array}$ & $\begin{array}{l}\text { - } \quad \text { Auto-reconocimiento y conciencia corporal } \\
\text { - } \quad \text { Exploración corporal: en el espacio, de } \\
\text { posturas, del gesto, de posibilidades de } \\
\text { desplazamiento y espacio-temporal } \\
\text { - Exploración de los sentimientos } \\
\text { - } \quad \text { Conciencia de estados (relajación - tensión) }\end{array}$ \\
\hline Comunicativa & $\begin{array}{l}\text { Comunicación no } \\
\text { verbal } \\
\text { Comunicación Oral } \\
\text { Comunicación escrita }\end{array}$ & $\begin{array}{ll}\text { - } & \text { Descubrir posibilidades de expresión de } \\
\text { sentimientos, emociones, pensamientos y } \\
\text { deseos } \\
\text { - } & \text { Representar } \\
\text { - } & \text { Recrear ambientes y momento } \\
\text { - } & \text { Utilizar medios no verbales y verbales }\end{array}$ \\
\hline Artística & $\begin{array}{l}\text { Las experiencias } \\
\text { plástica, musical } \\
\text { y teatral, c/u por } \\
\text { separado debe } \\
\text { realizarse desde los } \\
\text { siguientes } 4 \text { ejes: } \\
\text { - } \quad \text { Sensibilidad } \\
\text { - } \quad \text { Expresión } \\
\text { - } \quad \text { Creatividad } \\
\text { - } \quad \text { Sentido estético }\end{array}$ & $\begin{array}{l}\text { - Realizar movimientos corporales } \\
\text { - } \quad \text { Explorar y diferenciar formas colores, } \\
\text { formas y texturas, así como direcciones en } \\
\text { el espacio } \\
\text { - } \quad \text { Utilizar los sentidos en la vivencia } \\
\text { cotidiana } \\
\text { - Desarrollar paulatinamente la manualidad } \\
\text { - } \quad \text { Distinguir y apreciar algunos elementos de } \\
\text { las artes } \\
\text { - } \quad \text { Representar la figura humana, seres, } \\
\text { objetos y situaciones } \\
\text { - Expresarse usando diferentes técnicas, } \\
\text { incluso combinándolas } \\
\text { - Tener experiencias como autor } \\
\text { - } \quad \text { Analizar y generar preguntas }\end{array}$ \\
\hline
\end{tabular}




\begin{tabular}{|c|c|c|}
\hline Dimensiones & $\begin{array}{c}\text { Ejes - Campos de } \\
\text { trabajo }\end{array}$ & Desarrollos por fortalecer / a nivel general \\
\hline $\begin{array}{l}\text { Cognitiva } \\
\text { (Asimilación, } \\
\text { Acomodación, } \\
\text { Equilibrio, } \\
\text { Desequilibrio) }\end{array}$ & $\begin{array}{l}\text { Relación con : } \\
\text { - } \quad \text { la naturaleza } \\
\text { - } \quad \text { con grupos } \\
\text { humanos y } \\
\text { prácticas culturales } \\
\text { - } \quad \text { lógico matemática }\end{array}$ & $\begin{array}{l}\text {-Descubrir las posibilidades perceptivas de los } \\
\text { sentidos } \\
\text { - Relacionar sus experiencias con la } \\
\text { cotidianidad y su entorno } \\
\text { - Establecer secuencias y patrones; relaciones } \\
\text { espaciales y temporales; diferencias de } \\
\text { tamaño y características. }\end{array}$ \\
\hline
\end{tabular}

TABLA 4. Esquema general que resume y las relaciona las dimensiones, ejes y aspectos a desarrollar del Lineamiento Pedagógicos y Curriculares del Distrito del Bogotá, 2013.

En el esquema general del Lineamiento del Distrito (tabla 4), a simple vista resalta la centralidad de la actividad corporal en todas las dimensiones, ejes y desarrollos por fortalecer, verificándose la intención de integrar una concepción del cuerpo como vivencia activa y privilegiada en el preescolar. Sin embargo, al visualizar las correlaciones entre las dimensiones, ejes y aspectos a desarrollar planteados, se verifica que:

- Las dimensiones, hacen referencia a los aspectos de la dimensión humana, es decir aquella que el niño desarrolla en relación a sí mismo y con el entorno, siempre en una unicidad del ser (cuerpo-mente). Tal como están planteadas las cinco dimensiones, presentan un punto de confusión en el momento de diseñar una estrategia de aplicación, en cuanto indican una diferenciación entre lo personal y lo corporal, una dicotomía que no existe en la esfera afectiva del ser, y además consideran entre las dimensiones el "arte" cuando es un pilar metodológico.

- En los ejes o campos de trabajo, se encuentra que aquellos que hacen parte de la comunicación y la experiencia artística son implícitos en la expresión y la creatividad del cuerpo y el movimiento, así como en la relación con los grupos y prácticas culturales. Es decir, hay una estructura de campos de trabajo repetitiva que al momento de ser plasmada en actividades, puede generar una programación extensa y/o confusa.

- Los desarrollos por fortalecer se repiten en más de un eje sin considerar la lógica del aprendizaje global del niño.

La confusión y/o ausencia conceptual en lo referente al cuerpo y el movimiento es apenas analizada y también se refleja en el aula, como lo indica la investigación denominada, La Formación Docente en el trabajo con el cuerpo y el movimiento en Educación Inicial, desarrollada por María Consuelo Martín (2003), en algunas instituciones de Bogotá. Esta investigación permitió concluir, que el "cuerpo" en la etapa de preescolar del niño se concibe como un instrumento para la adquisición eficaz de los aprendizajes, hecho observable desde tres aspectos con los que se 
incorpora durante la realización de un trabajo: el manejo de posturas durante las actividades, el afianzamiento de la motricidad fina durante la elaboración de guías, y la realización de movimientos orientados por el maestro, encontrando además que los momentos donde los pequeños:

Logran hacer de su cuerpo un medio de expresión, comunicación y creación, ocurren especialmente en los descansos o cuando el adulto no está presente; -así como también- gracias a la acción pedagógica intencionada de un docente en situaciones como las propuestas en clase de expresión corporal o los momentos de bienvenida y despedida (Martín, 2013, p. 76).

Ante este panorama, se debe resaltar el esfuerzo de la SEB para para formar sus docentes de preescolar en didáctica, por medio de los cursos sobre la filosofía de Reggio Emilia, que se fundamenta en la pedagogía de la escucha, enfoque aplicativo de cambio y adaptación de los procesos educativos según el interés que el niño da a los proyectos propuestos, a partir de los cuales se desarrollan los conceptos y las prácticas por medio de la investigación de niños y maestros. Esta filosofía implica aspectos de la pedagogía Montessori y se adapta al proceso de descubrimiento natural del niño y su relación con el entorno, implicando esto al cuerpo, pero concretamente no considera la corporeidad, manteniéndose sobre una línea de desarrollo cognitivo y del lenguaje.

Una vez más, tomando el caso de Bogotá como referente de la realidad de la institución educativa del preescolar en Colombia, se deduce que el cuerpo y el movimiento se conciben de manera funcional y no vivencial. Funciones con fines primordialmente escolares de dos tipos: por una parte, preparar al niño para que sea un mejor alumno en una primaria mayormente académica, y por otra parte, con la función de producir la presentación de fin de año. En ambos casos se desconoce el valor de la corporeidad y del movimiento en preescolar, el valor de permitir y favorecer el autoconocimiento, autocuidado y reconocimiento de sí mismo, para que crezca un ciudadano feliz con el pleno desarrollo de sus capacidades, sensitivas, emocionales y relacionales. Casi se podría decir que en el preescolar, tanto público como privado, se da prioridad al desarrollo intelectual al trabajar con muchas actividades de mesa y enviando a casa tareas de tipo conceptual desde una tierna edad, dejando en segundo plano el desarrollo emocional y de personalidad que debiera ser objetivo central de la educación de la primera infancia.

\section{Análisis integrador de las teorías de cuerpo y movimiento en relación al contexto colombiano}

Casi cuarenta años después de los estudios de Vayer y Destrooper sobre la inadaptabilidad social del niño, se podría decir que las cosas no han cambiado mucho. Podemos ver hoy (2014), que las dificultades que el niño encuentra en su 
experiencia cotidiana continúan siendo muchas y siempre ligadas a la comunicación en diferentes niveles y ámbitos. Siguen siendo las dificultades más evidentes, el paso de la vida indiscutiblemente más veloz y la influencia que la difusión de la tecnología tiene sobre nuestra manera de percibir y vivir la realidad, determinando de un lado, que la visión y el oído son los sentidos más desarrollados y del otro, que el conocimiento sea transmitido de modo más inmediato por medio de imágenes. En consecuencia y en relación a las teorías sociales de la educación (Vygotsky 1979, 1982a, 1982b), Bloom (1967), Denis (1980), Lewis (1982) y Montessori (1987, en Gil-Madrona et al, 2008) se debe considerar la influencia que la televisión, internet y los diferentes dispositivos tienen, no solo en relación al entretenimiento o al conocimiento, sino sobre todo en la calidad del tiempo dedicado a relacionarnos con la familia, los otros y la realidad circunstante, así como también podemos observar que en diversas ocasiones, el niño transcurre su jornada entre la escuela y cursos extraescolares o terapias, viniéndole a faltar la relación afectiva fundamental de tiempo con los padres.

Aunque en los últimos años, tanto en política pública educativa como en las instituciones del estado (SED, MEN, SDIS y Alcaldía de Bogotá) y las facultades de educación han mostrado una gran apertura respecto a la integración del conocimiento científico del desarrollo en los primeros 6 años de vida, como parte de las propuestas educativas de preescolar. Aún hoy podemos observar que falta un estudio real sobre la didáctica para una educación que tenga en cuenta la corporeidad y la gran necesidad de movimiento que tiene el niño. En las directrices colombianas para preescolar y en la reglamentación institucional siempre se indica el "cuerpo y el movimiento", pero sin especificar una didáctica precisa o legislar la presencia de un profesional de la materia en preescolar, y es en el aspecto práctico-operativo que se desarrolla en la institución educativa que se concreta la formación del futuro ciudadano.

Respecto al Lineamiento del Distrito de Bogotá, analizado como espejo de la realidad nacional (tabla 4), encontramos que brinda una orientación didáctica para el preescolar por medio de una indicación clara de los objetivos a que se debe llegar en cada desarrollo por fortalecer, así como también indica las prácticas o pilares para desarrollar en el aula. Sin embargo, no estructura correctamente las dimensiones del ser y por ello no puede organizar una secuencialidad de los ejes o campos de trabajo a considerar, generando una gran complejidad en los desarrollos por fortalecer, sobre los cuales se estructura la programación a desarrollar. Para poner en práctica el Lineamiento tal como está indicado, sería necesario plantear muchas actividades por cada eje, y de esta manera la programación no se estructuraría con la concepción global con la que el niño llega a su proceso cognitivo. Debemos tener en cuenta también, que el docente de preescolar no siempre se siente en grado de desarrollar experiencias de música, teatro, danza, arte, etc., considerando además, que en muchos preescolares no se cuenta con un cierto número de docentes especializados para realizarlas. 


\section{Conclusiones}

Siendo ampliamente explicado por modernos estudios de neurología que en primera infancia ( 0 a 6 años), el movimiento constituye el principal medio del hombre para interactuar con los demás y con el entorno. El correcto desarrollo de la corporalidad influye en un desarrollo emocional positivo, así como en el aprendizaje relacional y conceptual, se confirma la unicidad cuerpo-mente y se concluye que "la experiencia fundamental de vivir es la comunicación que se establece con el mundo a través del cuerpo que experimenta, conoce, dialoga e investiga. En consecuencia, el movimiento como actividad física es la experiencia de vida y constituye la práctica de la deducción de la fase operativa, íntimamente ligada a la acción del medio ambiente circundante" (Gutiérrez, 2013).

Desde esta perspectiva, una experiencia educativa no sedentaria, dirigida a la exploración experiencial en el espacio, con los objetos en actividades que invitan a las relaciones será el camino para el pleno desarrollo de los niños en preescolar. Para ello, es indispensable la claridad conceptual del Lineamiento en cuanto a: las dimensiones del ser, los aspectos concretos sobre los cuales realizar la experiencia que llevará al conocimiento, y la definición de una didáctica que incluya temáticas globales de aprendizaje y guías de realización que oriente al docente sobre como estructurar la cotidianidad en el aula de preescolar. Buscando esta claridad conceptual, se plantea una concepción de las dimensiones del ser (tabla 5), indicando solo las tres en los cuales se evoluciona (psicofísico, socio-relacional y cognitivo) y relacionándolas con la forma de aprendizaje global del niño, en la cual se identifican tres experiencias concretas con los cuales se llega al conocimiento (corporal, comunicativo y representativo), denominados ejes o campos.

\begin{tabular}{|c|c|c|}
\hline Dimensiones & $\begin{array}{l}\text { Ejes/Campos de } \\
\text { trabajo }\end{array}$ & $\begin{array}{l}\text { Desarrollos por fortalecer / } \\
\text { a nivel general }\end{array}$ \\
\hline \multirow[t]{3}{*}{ Psico-física } & Corporal & $\begin{array}{l}\text { - Imagen, percepción y conocimiento } \\
\text { - Auto-reconocimiento y conciencia corporal } \\
\text { - Exploración de los sentimientos } \\
\text { - Conciencia de estados (relajación - tensión) } \\
\text { - Descubrir las posibilidades perceptivas de los } \\
\text { sentidos }\end{array}$ \\
\hline & Comunicativo & $\begin{array}{l}\text { - Descubrir posibilidades de expresión de } \\
\text { sentimientos, emociones, pensamientos y deseos }\end{array}$ \\
\hline & Representativo* & $\begin{array}{l}\text { - Adoptar posturas } \\
\text { - Representar la figura humana. }\end{array}$ \\
\hline
\end{tabular}


Dimensiones

\section{Socio-cultural}

\section{Corporal -Movi- miento como medio de interacción}

Representativo y
sentido estético*

\section{Desarrollos por fortalecer / a nivel general}

- Relación con la Naturaleza (pre-ciencias)

- Relación con grupos humanos y prácticas culturales (pre-sociales)

- Relaciones lógico Matemática (pre-matemática)

- Analizar y generar preguntas

- Relacionar sus experiencias con la cotidianidad y su entorno

- Identificarse y relacionarse con sus pares

- Representar seres, objetos y situaciones (preciencias)

- Recrear ambientes y momentos (pre-sociales)

- Utilizar medios no verbales y verbales

- Tener contacto con diferentes materiales (preciencias)

- Disfrutar de las actividades

- Distinguir y apreciar algunos elementos de las artes

- Realizar movimientos corporales

- Exploración en el espacio de posturas y (relación espacio-temporal (pre-matemática)

- Establecer secuencias y patrones; diferencias de tamaño y características (pre-matemática).

- Comunicación no verbal - relacionada con representativo

- Comunicación oral y lenguaje: lecturas

- Comunicación escrita - símbolo y pre-escritura

- Explorar y diferenciar formas colores, formas y texturas, así como direcciones en el espacio (pre-matemática)

Representativo* - Desarrollar paulatinamente la manualidad

- Expresarse usando diferentes técnicas, incluso combinándolas

- Tener experiencias como autor

* Integra las experiencias plástica, musical y teatral; cada una para proveer el desarrollo de la sensibilidad, la expresión y la creatividad

TABLA 5. Propuesta de reorganización de dimensiones, ejes y aspectos a desarrollar del Lineamiento Pedagógico y Curricular del Distrito del Bogotá - 2013. 
La relación entre dimensiones del ser y ejes del conocimiento aquí propuesta (tabla 5), permite agrupar los desarrollos por fortalecer o los objetivos a lograr de manera correlacionada y no repetitiva, facilitando así, identificar al momento de realizar una programación, que tipo de experiencia indicada en los pilares (juego, arte, literatura o exploración del medio) será las más adecuada. Así mismo, permitirá proporcionar el conocimiento de manera multidisciplinar, al integrar en cada actividad objetivos de diversos saberes y conceptos, a través de la didáctica indicada en los pilares, entendiéndolos como experiencias lúdicas y no como producciones técnicas.

En el plano aplicativo en preescolar, la inclusión de la corporeidad y su vivencia activa por medio del movimiento, entendida como un lenguaje que brinda información diaria e importante a los niños, y que juega un papel importante, dando los estímulos para que el aprendizaje con raíces en el descubrimiento y la experimentación se concrete en una programación didáctica que apunta a la educación global, con la cual se evita trabajar por materias o áreas, por ser un sistema académico no adecuado al niño que conlleva al sedentarismo y al continuo trabajo de mesa. En la educación global se trabaja por temáticas o proyectos, que implican una serie de experiencias llenas de significado para el niño, con las cuales alcanzar los diferentes objetivos de la Educación de la Primera Infancia, como se evidencia en la guía para estructurar la planeación de cada tema o proyecto (figura 2). Este tipo de planeación es adapta a preescolar, porque permite la realización de actividades que involucran múltiples experiencias y objetivos, permitiendo completar plenamente una programación anual en la cual los rituales (rutinas diarias) del preescolar como la bienvenida, los momentos de alimentación, el juego libre, descanso pueden ser integrados en la programación como momentos de aprendizaje, según el argumento a desarrollar. 


\section{TEMA o PROYECTO:}

Actividad motivante: Una motivación fuerte (un juego, una sorpresa, la experiencia de observación) Duración: (número de sesiones, jornadas, días - se puede indicar por fechas)

\begin{tabular}{|c|c|c|}
\hline $\begin{array}{l}\text { Aspectos que } \\
\text { debe implicar }\end{array}$ & $\begin{array}{c}\text { Objetivo según tema o } \\
\text { proyecto }\end{array}$ & Actividades cognitivas según temática* \\
\hline $\begin{array}{l}\text { Psico-físico: } \\
\text { Corporeidad y } \\
\text { auto-cuidado }\end{array}$ & $\begin{array}{l}\text { - Favorecer el reconocimiento } \\
\text { de sí mismo y del otro. } \\
\text { - Propiciar el conocimiento y } \\
\text { consolidación del esquema } \\
\text { corporal. } \\
\text { - Incentivar hábitos de } \\
\text { auto cuidado en higiene, } \\
\text { alimentación y descanso. }\end{array}$ & $\begin{array}{l}\text { Actividades que permitan: } \\
\text { 1. El contacto con sí mismo como masajes, } \\
\text { juegos con el espejo. } \\
\text { 2. Exploración de los sentimientos como } \\
\text { juegos de roles, teatro, expresión corporal. } \\
\text { 3. Conciencia de estados (relajación/tensión) } \\
\text { 4. Explorar los sentidos - Texturas, masas, } \\
\text { sabores. }\end{array}$ \\
\hline $\begin{array}{l}\text { Socio-cultural: } \\
\text { Comunicación, } \\
\text { Integración y } \\
\text { Participación. }\end{array}$ & $\begin{array}{l}\text { - Estimular la participación e } \\
\text { integración del niño durante } \\
\text { las experiencias. } \\
\text { - Estimular las habilidades } \\
\text { personales de comunicación y } \\
\text { de expresión. } \\
\text { - Reconocer y expresar los } \\
\text { propios sentimientos y } \\
\text { emociones. } \\
\text { - Desarrollar la capacidad } \\
\text { de atención, comprensión } \\
\text { y escucha del otro y del } \\
\text { entorno. }\end{array}$ & $\begin{array}{l}\text { Momentos de verbalización de sentimientos, } \\
\text { sensaciones e ideas del niño, de manera } \\
\text { individual y/o colectiva; y toda forma de } \\
\text { incentivar la participación y la creación en } \\
\text { cada momento de la jornada. } \\
\text { Momentos literarios con lecturas cortas y/o } \\
\text { narraciones; conversaciones, entre otras. } \\
\text { Interpretación de canciones y juegos de roles. } \\
\text { Dramatizaciones, encuentros, entre otros. }\end{array}$ \\
\hline $\begin{array}{l}\text { Movimiento y } \\
\text { Motricidad }\end{array}$ & $\begin{array}{l}\text { - Desarrollar las habilidades } \\
\text { motrices gruesas y } \\
\text { conocimiento del entorno. }\end{array}$ & $\begin{array}{l}\text { Realización de actividades motoras guiadas y } \\
\text { libres, con o sin objetos. } \\
\text { Danza, teatro, Psicomotricidad, entre otros; } \\
\text { donde debe prevalecer la creatividad, la libre } \\
\text { expresión y la imaginación. }\end{array}$ \\
\hline Motricidad fina & $\begin{array}{l}\text { - Desarrollar actividades } \\
\text { manuales y gráficas estimular } \\
\text { las diversas manipulaciones } \\
\text { que estimulan la motricidad } \\
\text { fina y la apreciación estética. }\end{array}$ & $\begin{array}{l}\text { Realización de actividades como diseñar, } \\
\text { colorear, construir, ensartar, amasar, entre } \\
\text { otros. }\end{array}$ \\
\hline \multicolumn{3}{|c|}{$\begin{array}{l}\text { Indicar la interrelación con campos de experiencia: áreas y temas con que se genera el conoci- } \\
\text { miento transversal, aquí se indica todo el conocimiento cognitivo a alcanzar. Ejemplo: }\end{array}$} \\
\hline \multicolumn{3}{|c|}{$\begin{array}{l}\text { Pre matemática (colores, formas, ubicación espacial); } \\
\text { Ciencias (animales, eventos naturales, clima, el cuerpo; así como experimentos, entre muchos) } \\
\text { Lenguaje (poesías, narraciones, historias) } \\
\text { Artes plásticas (dibujos, esculturas, collage, entre otros) } \\
\text { Artes Escénicas_(juegos de roles, dramatizaciones, entre otros) } \\
\text { Música. }\end{array}$} \\
\hline
\end{tabular}

FIGURA 2 Ficha modelo para estructurar la planeación de cada tema o proyecto de aula en preescolar (en relación a la propuesta de la tabla 5). 
Específicamente para el desarrollo de las actividades de Corporeidad y movimiento se propone considerar, La Expresión Corporal de Patricia Stokoe (1979; 1984), para el jardín de infantes basada en el movimiento libre para explorar la música y el espacio, y la propuesta de actividad motriz, Espacios de Acción y Aventura de Mendiara, J (1999) que se basa en el desarrollo de la movilidad del niño, mediante historias inventadas que a la vez también desarrollan su creatividad, y la cual invita a generar la exploración del espacio dependiendo de la historia. Como consecuencia de lo apenas propuesto, el educador podrá descargar en la actividad y no en sí mismo la atención del niño mejorando el desempeño de su quehacer teniendo en una dinámica pedagógica que favorece la creación, la autorregulación y la toma de decisiones que favorece el conocimiento. 


\section{Referencias}

Baldasarre, B. (Eds). (2006). Inizziazione alla pedagoga artistica, conoscersi para educar. Roma: Edizioni iniziación mediterranea.

Baril, J. (1987). La Danza moderna. Barcelona: Paidós .

Biferale, S. (Eds). (2009). L'apprendimento musicale tra senso e sensorialità. Ponencia presentada en el $1^{\circ}$ Convegno II bambino in ascolto. Realizada por la Associazione italiana Gordon per lo sviluppo musicale - AIGAM. Roma 4-5 dicembre.

Cacciavillani, M. (2009). Sviluppo motorio e plastica neurale. Ponencia presentada en el $1^{\circ}$ Convegno II bambino in ascolto. Realizada por la Associazione italiana Gordon per lo sviluppo musicale - AIGAM. Roma 4-5 dicembre.

Cachadiña, C.. (2004.) Expresión corporal y creatividad: métodos y procesos para la construcción de un lenguaje integral. Madrid: Instituto Nacional de educación física, Universidad politécnica de Madrid.

Castro V. (2013). La conveniencia de un argumento pedagógico a propósito de una política pública en educación inicial y primera infancia, Artículo Editorial. Revista Educación y Ciudad. 24, 7-8.

Copola, L. (2010). Rudolf von Laban y la danza libre. Recuperado de www.giornalede ladanza. com. Consultado en junio 2012.

Gallavotti, Barbara. (Eds). (1999). Il corpo umano: strategie di una macchina perfetta. Milán: Mondadori.

Gil-Madrona, P. Contreras, O. González, S. y Gómez, I. (2008) "Justificación de la educación física en la educación infantil. Educación y Educadores. (11)2, 159-177. Recuperado en http://www.redalyc.org/pdf/834/83411211.pdf

Gubbay, My Kalmar, D. (1985). SENSOPERCEPCION. Revista Uno Mismo. Recuperado de http:// kalmarstokoe.com.ar/articulos-conferencias-publicaciones/sensopercepcion-marina-gubbayy-deborah-kalmar. Consultado en 2010.

Gutiérrez, M. (2013) La expresión corporal como estrategia educativa para la enseñanzaaprendizaje de un L2 a niños de 4 a 5 años. Tesis de Maestría en Educación. Universidad Tec-Virtula, Tecnológico de Monterrey, México.

Kalmar, D. (2003). Con los ojos del Corazón: Aportaciones principales de la pedagogía de Patricia Stokoe. Ponencia presentada en el Primer Congreso Internacional de Expresión Corporal del Departamento de Didáctica de la Expresión Músical, Plástica y Corporal de la Universidad de Salamanca junto con la Asociación AFYEC. Recuperado de http:// kalmarstokoe.com.ar/articulos-conferencias-publicaciones/sensopercepcion-marinagubbay-y-deborah-kalmar Consultado en junio 2010

Langlade, A., Rey de Langlade, N. (Eds). (1986). Teoría general de la gimnasia. Buenos Aires: Editorial Stadium.

Lapierre, A. (Eds). (2001). Dalla psicomotricità relazionale all'analisi corporea della relazione. Roma: Armando. 
Le Boulch, J. (Eds). (1983). El desarrollo psicomotor desde el nacimiento a los 6 años. Madrid: Doñate.

Le Boulch, J. (Eds). (1981). La educación por el movimiento en la edad escolar. Barcelona: Paidós Ibérica.

Maganto, C. (1998). Evaluación del desarrollo sensoriomotor. En Bueda-Casal y Sierra, J. Manual de Evaluación Psicológica. Fundamentos, técnicas y aplicaciones. Madrid: Siglo XXI.

Martin C.,. (2013). La Formación Docente en el trabajo con el cuerpo y el movimiento en Educación Inicial. Revista Educación y Ciudad. (24): 73-79.

Mendiara Rivas, J. (1999) Espacios de acción y aventura. Apuntes,.(56): 65-70.

Ministerios de Protección Social y Educación e Instituto colombiano de Bienestar Familiar. (2007). Política Pública Nacional de la Primera Infancia "COLOMBIA POR LA PRIMERA INFANCIA". Bogotá: Ministerio de Educación Nacional.

Ormrod, J. (Eds). (2008). Aprendizaje humano. Madrid: Pearson/Pretince Hal.

Puyuelo M. (1998). Logopedia y parálisis cerebral En Peña, J. Manual de Logopedia. (1ª Ed.) Barcelona: Masson.

Secretaria de Integración Social y Alcaldía Mayor de Bogotá. (2013). Lineamiento Pedagógico y Curricular para la educación inicial del Distrito. Bogotá: DVO universal.

Stokoe, P. et. al. (Eds). (1984). La expresión corporal en el Jardín de infantes. Buenos Aires: Paidos.

Stokoe, P. (1979). Expresión corporal En Germani C. et.al. (Eds.) Teoria de la educacón prescolar. Buenos Aires: Eudeba.

Vayer, P. (Eds). (1973). L'educazione psicomotoria in etá prescolastica. Colana medico pedagogica. Roma, Italia: Armando Editori (tradotto dal franzase).

Vayer, P., Destrooper, J. (Eds). (1976). Il corpo nella Dinamica Educativa: il disadattamento come paralisi della comunicazione. Titolo originale: La dymnamique de l'action éducative chez les enfants inadaptés. Paris: Doin Editeurs,. In Itlaiano: Milano: Emme Edizione.

Vernia M. (2012). Método pedagógico musical Dalcroze. Revista Artseduca, 1. Recuperado de http://www.artseduca.com. Consultado en abril 2014.

Zamorano M. (2010). La Expresión Corporal en el siglo XX. Magisterio y educación física Universidad complutense, apuntes. Recuperado de: mefuc.files.wordpress. com/2010/02/2-9.pdf Consultado el mayo 2014.

Recibido: 30 mayo 2013

Aceptado: 31 julio 2013

Como citar:

Gutiérrez, E., Castillo, J.A. (2014). Reflexiones sobre la concepción del cuerpo y del movimiento para una educación integral de la primera infancia. Praxis Pedagógica, 15, 15-42 